\title{
Broadband interference filters with suppression of high reflection bands of 4-, 5- and 6-th orders
}

\author{
V.Yu. Pervak, L.V. Poperenko \\ Taras Schevchenko Kyiv National University, Physics Department \\ 6, prospect Academician Glushkov, 03022 Kyiv, Ukraine \\ E-mail:pervak@univ.kiev.ua
}

\begin{abstract}
The influence of optical constants dispersion on spectral properties of multilayer interference systems, in which spectral characteristics suppression of the adjacent bands of high reflection of 4,5 and 6-th orders are observed, is investigated. Examples of realization of synthesized interference filters are resulted and features of their manufacturing are considered.
\end{abstract}

Keywords: wideband interference filter, suppress of high reflection bands.

Manuscript received 25.05.05; accepted for publication 25.10.05.

\section{Introduction}

It is known that multilayer periodic interference systems formed by replication of the periods $\mathrm{AB}[1,2], \mathrm{ABCBA}$ [3, 4], ABCCBA or ABCDDCBA [5-7] where A, B, C, and $\mathrm{D}$ layers of equal optical thickness from materials with refractive indecies $n_{A}, n_{B}, n_{C}$ and $n_{D}$, accordingly, are characterized by spectral dependences of transmission with alternating bands of high reflection and transsmision. Systems $(\mathrm{AB})^{p}$ where $p$ is the number of the periods are characterized by spectral dependences of transsmision in which for wavelengths $\lambda$ with odd integer values of the relative wavelength $\lambda_{0} / \lambda=1,3,5 \ldots$ the high reflection bands are separated one from another by transmission bands [1]. Thus, the optical thickness of the period is equal to half of the fixed wavelength $\lambda_{0}$. Integer values of relative wavelength $\lambda_{0} / \lambda$, usually name the order of a band. In a scale of relative wavelengths $\lambda_{0} / \lambda$ the centers of transmission bands coincide with even integer values of the ratio $\lambda_{0} / \lambda=2,4,6 \ldots$ The relative width of high reflection bands and transmission is determined by a difference of refractive indecies $n_{A}$ and $n_{B}$, and reflection in a high reflection bands by a number of the periods. At the fixed values $n_{A}$ and $n_{B}$, and also preservation of the optical thickness of the period equal $\lambda_{0} / 2$, deviations of optical thickness of layers $A$ and $\mathrm{B}$ from $\lambda_{0} / 4$ result in narrowing high reflection bands and corresponding expansion of transmission bands [2]. At any values $n_{A}$ and $n_{B}$ transmission widths $\Delta\left(\lambda_{0} / \lambda\right)$ in units of $\lambda_{0} / \lambda$ cannot be larger than 2 , if the high reflection band of the first order is observed [3].
The transmission bands of structures $(\mathrm{ABCBA})^{p}$ may be widened by 1.5 times [3, 4], and structures $(\mathrm{ABCCBA})^{p}$ and $(\mathrm{ABCDDCBA})^{p}$ [5-7] by 2 and 3 times accordingly, in comparison with those of structures $(\mathrm{AB})^{p}$. The optical thickness of all the layers in the period should be equal. The widening of the transmission bands of such systems occurs due to suppression of adjacent bands of high reflection. In the case of periods ABCCBA, the suppression of three adjacent high reflection bands (from the 2-nd to the 4-th orders) is possible. In the case of periods ABCDDCBA, the suppression of five adjacent high reflection bands (from the 2-nd to the 6-th orders) is possible. However, the suppression of corresponding high reflection bands is achievable at rather strict performance of the relations that describe interrelation of refractive indecies of separate layers in the periods. Besides, all the layers in the systems are considered to be not absorbing, and the dispersion of refractive indecies is not taken into account. In such approximation, the spectral dependences of transmission are described by periodic functions, the period of which in units of the phase thickness is equal to $v \sum \varphi=v \pi$, which in units of the relative wavelength $\lambda_{0} / \lambda$ corresponds to the range from 0 to $v . \sum \varphi$ is the total phase thickness of the symmetric period for $\lambda=\lambda_{0}$, and $\varphi=(2 \pi / \lambda) n d$ is the phase thickness of the layer, $n d=\left(\lambda_{0} / 2 v\right)$ is the optical thickness of the layer, $v$ is the number of layers. In $[3,5]$, the rule was formulated according to which the total number of suppressed high reflection bands in the 
spectral range from 0 to $v$ on a scale of relative wavelengths is determined by the number of layers in the symmetric period and cannot be greater than $[(v-2) / 2+1]$ for even $v$ and $[(v-1) / 2+1]$ for odd $v$. Keeping the component of the period to be constant (amount of various materials), the change of the total number of layers in the period is possible to achieve various position in spectra of the widened transmission bands [8-10]. Thus, the number of adjacent suppressed high reflection bands cannot be greater than $(2 m-3)$ where $m$ is the number of the various film-forming materials used for creation of the multilayer periodic structure with the symmetric periods, and the maximal number of adjacent suppressed high reflection bands is achieved in that case, when the number of layers with the same optical thickness into which it is conditionally possible to break the period, will be even. The order of the central suppressed high reflection band is twice less than the number of layers in the period with layers of the equal optical thickness.

However, used at synthesis of the multilayered structures considered above, the assumption of absence of refractive index dispersion and a very low absorption factor for separate layers is more often difficult to realize in practice. If the operation spectral range of a multilayer structure includes not only the range of the transmission band and also the range of high reflection bands of various orders, the wavelengths of the beginning and the end of the operation range differ sometimes by 5 to 8 times. In this case, the neglect is unlikely justified by refractive index dispersion, and therefore ratio between refractive indecies in various ranges may be different. Taking into account that layer refractive indecies should correspond strictly to the certain ratio over the whole operation spectral range, there are significant difficulties with the choice of layer materials.

In the work, by the example of multilayer structures in which wide transmission bands are formed by suppression of high reflection bands of 4-th, 5-th and 6-th orders, shown are the ways to optimum choose of materials and parameters of the structure for elimination of undesirable influence of optical constant dispersion on filter spectral characteristics.

The suppression of high reflection bands of 4-th, 5-th and 6-th orders is achieved in multilayer structures with the three-component 10-layer symmetric period. At preservation of high reflection band of the first order, only four variants of the period structure are possible [9]:

ABCCCCCCBA,

AABCCCCBAA,

ABBBCCBBBA,

\section{AAABCCBAAA,}

where $A, B$ and $C$ are layers of materials with the refractive indecies $n_{A}, n_{B}$ and $n_{C}$, respectively, and identical optical thicknesses, equal to $0.05 \lambda_{0}$.

The refractive indecies $n_{A}, n_{B}$ and $n_{C}$ should satisfy the ratio:

$$
\begin{aligned}
& y=-0.15362+0.996 x+0.37678 x^{2}+0.03826 x^{3} \\
& \text { for the period }(1), \\
& y=0.004+0.73886 x \text { for the period }(2), \\
& y=-0.08796+1.16933 x-0.10692 x^{2}+0.03113 x^{3} \\
& \text { for the period }(3), \\
& y=0.22201+0.98432 x-0.24288 x^{2}+0.039416 x^{3}
\end{aligned}
$$$$
\text { for the period (4), }
$$

where $x=n_{B} / n_{C}$ and $\mathrm{y}=n_{A} / n_{B}$.

Proceeding from the necessity to achieve the greatest values of reflection in the range of high reflection and smoothness of transmission in the range of the transmission band, it is necessary to choose layer materials so that $n_{A}<n_{B}<n_{C}, n_{A}$ was as small as possible, and $n_{C}$ is as much as possible [9]. These conditions are satisfied with $n_{A}=1.45$ and $n_{C}=2.4$. Then, according to (5) (6) we shall receive for the periods (1) $n_{B}=1.98$, for the periods (2) $n_{B}=1.85$, for the periods (3) $n_{B}=1.86$, for the periods (4) $n_{B}=1.75$ [11-14]. The transmission spectra of multilayer structures with the periods (1)-(4) on substrates from fused quartz are shown in Fig. 1. The spectral characteristics of multilayer systems with the periods (1) and (4) are very similar. In the spectrum, the high reflection bands of the 1-st, 2-nd and 3-rd orders are observed. In units of relative wavelengths, the band of the 2-nd order is the widest one, and the band of the 3 -rd order is the narrowest one. A little less similar spectra of multilayer systems with the periods (2) and (3) are among themselves. In the spectra, the high reflection bands of the 2-nd, 4-th, 5-th and 6-th orders are suppressed, and high reflection bands of the 1-st and 3 -rd orders are observed. And for the period (2) wider are high reflection band of the 1-st order, and for the period (3) - the band of the 3-rd order.

\section{The influence of reflection index dispersion}

Among known film-forming materials, $\mathrm{TiO}_{2}, \operatorname{Pr}_{6} \mathrm{O}_{11}$, $\mathrm{ThO}_{2}$ and $\mathrm{SiO}_{2}$ oxides possess the mentioned above refractive indecies. In the range of $500-550 \mathrm{~nm}$, the films of these oxides have refractive indecies $2.4 ; 1.85$; 1.75 and 1.45 , accordingly. Depending on the type of technological process (electron beam, magnetron, ionic or electron beam with ionic support) of film deposition and separate parameters (temperature of a substrate, evaporation rate, residual pressure in the vacuum chamber, fractional pressure of oxygen) the refractive indecies of films can differ for the same material by 0.1 to 0.3 . Therefore, it is expedient to specify the film characteristics obtained using a specific equipment, before manufacturing the filters. The multilayer structures prepared in this work have been made by the method of electron beam evaporation in high vacuum on vacuum installation VU-2M (Belarussia). The optical constants of films were determined by numerical methods of approximation by using transmission spectra of single-layer films on silica substrates. Thus, 

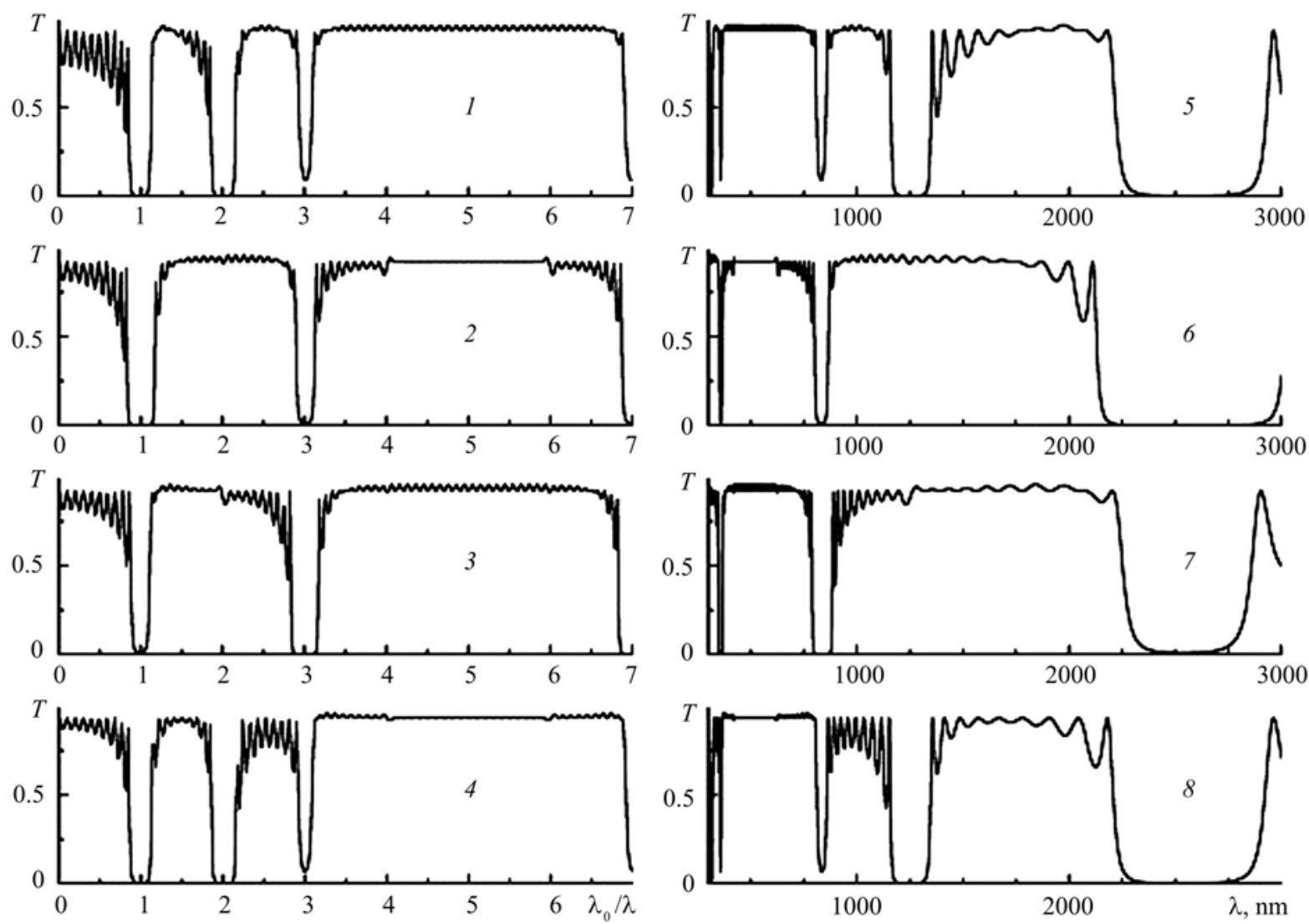

Fig. 1. Spectral dependences of the multilayer structure transmission $\mathrm{S}(\mathrm{ABCCCCCCBA}){ }^{10} \mathrm{~S}_{0}-1,5 ; \mathrm{S}(\mathrm{AABCCCCBAA}){ }^{10} \mathrm{~S}_{0}-2$, 6; $\mathrm{S}(\mathrm{ABBBCCBBBA}){ }^{10} \mathrm{~S}_{0}-3,7 ; \mathrm{S}(\mathrm{AAABCCBAAA}){ }^{10} \mathrm{~S}_{0}-4,8 . n_{A}=1.45 ; n_{C}=2.40 ; n_{S}=1.45 ; n_{S 0}=1.0 ; \lambda_{0}=2500 \mathrm{~nm}$; $n_{B}=1.98-1,5 ; n_{B}=1.85-2,3,6,7 ; n_{B}=1.75-4,8$.

technological modes of deposition were specified so that refractive indecies of obtained films were close to required for the wavelength $500 \mathrm{~nm}$ (the middle of the created transmission band). In Fig. 2, spectral dependences of film optical constants for the spectral range where the dispersion is the most essential are shown. In comparison with the dispersion of optical constants for $\mathrm{TiO}_{2}$ film, the dispersion for films of others oxides can be neglected, it is especial in the case of $\mathrm{SiO}_{2}$ layers. The extinction $k$ in $\mathrm{SiO}_{2}$ layers was no more than $10^{-5}$ and is not shown in the figure.

Uniqueness of researched multilayer structures consists of the fact that they can be realized with high accuracy by minimizing the mistakes in the thickness of deposited layers. To control the optical quality of deposited layers, we used monitoring of the thickness in the course of deposition. If as a control plate one uses the substrate with $\mathrm{TiO}_{2}$ layer (optical thickness $\lambda_{\mathrm{c}} / 4$, $\lambda_{\mathrm{c}}=500 \mathrm{~nm}$ ) preliminary deposited on it, formation of each layer begins and comes to the end at the moment of passage through the extreme value of transmission. Under deposition, the picture of signal changes repeats each subsequent period. However, in the case of a mistake of deposition of any layer, the process of the further optical control is necessary for correction.
Therefore, it is expedient to use at deposition of layers some control plates, on each of which to deposit no more than two periods. Fig. 3 shows the transmission change of the control plate when depositing first two and the last periods of multilayer structure, including the additional layer of $\mathrm{TiO}_{2}$. For the first period, the moments of the beginning and the termination of deposition of separate layers are specified.
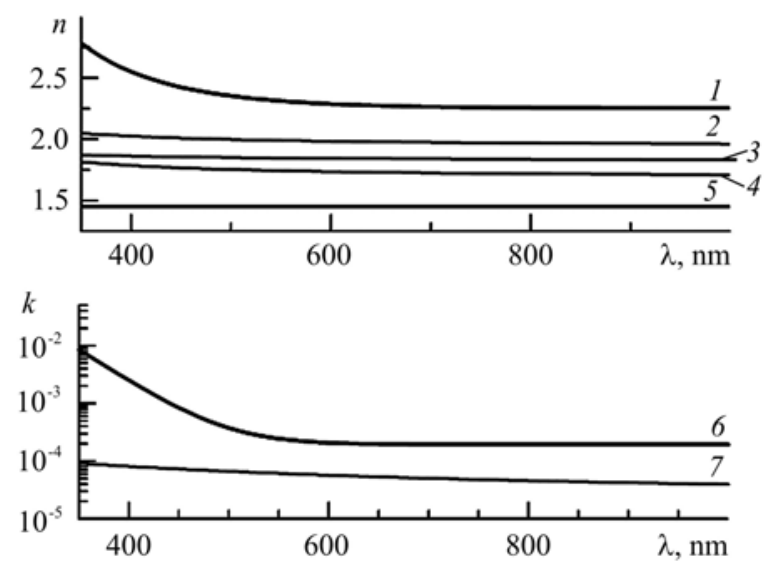

Fig. 2. Optical constants of layers: $\mathrm{TiO}_{2}(1,6), \mathrm{HfO}_{2}(2,7)$, $\mathrm{Pr}_{6} \mathrm{O}_{11}(3,7), \mathrm{ThO}_{2}(4,7)$ and $\mathrm{SiO}_{2}(5)$. 

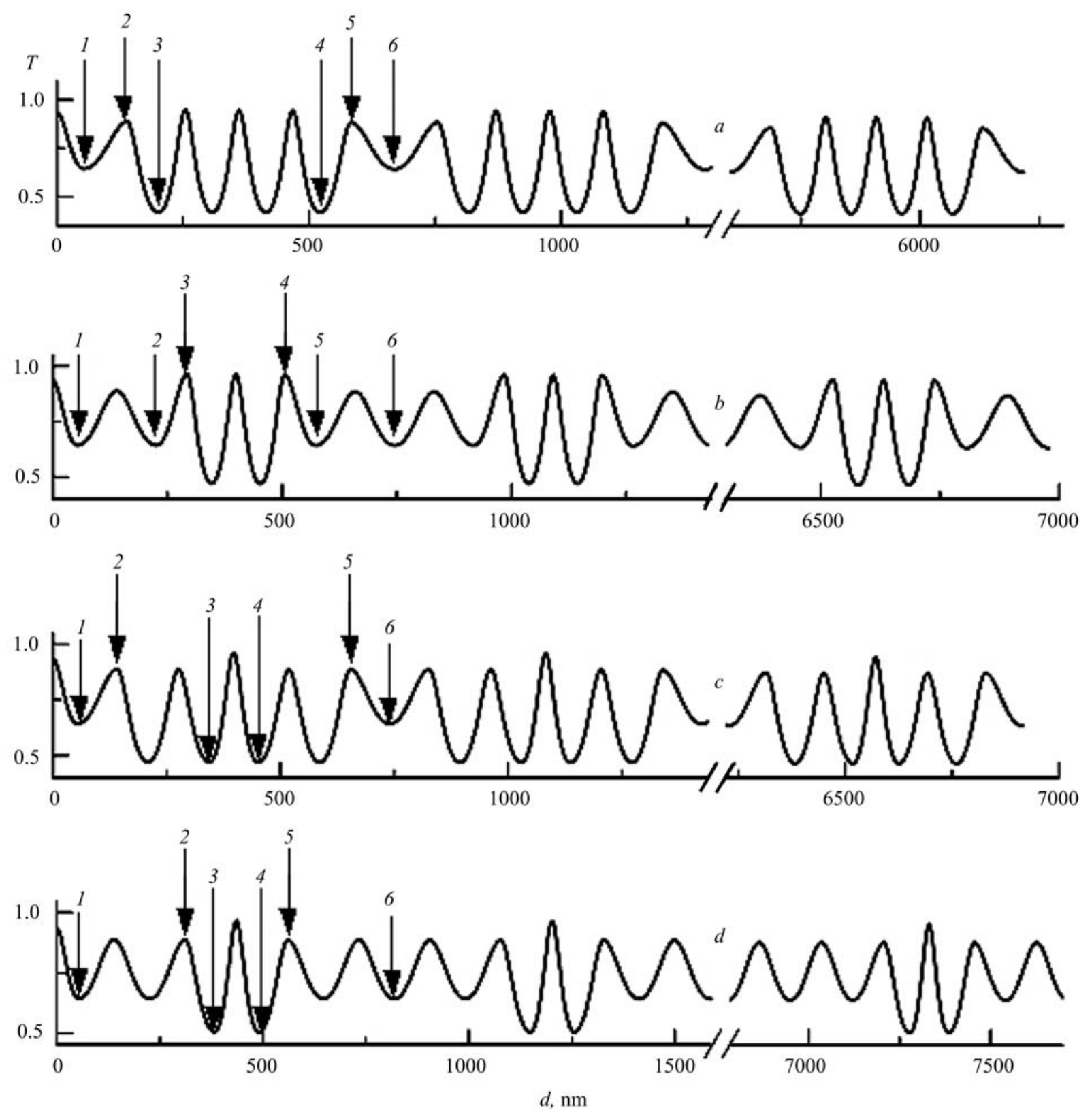

Fig. 3. Thickness dependence of the transmission of the coating for multilayer systems at the control wavelength $\lambda_{\mathrm{c}}=500 \mathrm{~nm}$ : $\mathrm{a}-\mathrm{S}(\mathrm{ABCCCCCCBA}){ }^{10} \mathrm{~S}_{0} ; \mathrm{b}-\mathrm{S}(\mathrm{AABCCCCBAA}){ }^{10} \mathrm{~S}_{0} ; \mathrm{c}-\mathrm{S}($ ABBBCCBBBA $){ }^{10} \mathrm{~S}_{0} ; \mathrm{d}-\mathrm{S}\left(\right.$ AAABCCBAAA) ${ }^{10} \mathrm{~S}_{0}$. A, B, C layers with the optical thickness $\lambda_{\mathrm{c}} / 4$ from $\mathrm{SiO}_{2}, \mathrm{HfO}_{2}, \mathrm{TiO}_{2}$ (a); $\mathrm{SiO}_{2}, \mathrm{Pr}_{6} \mathrm{O}_{11}, \mathrm{TiO}_{2}$ (b, c); $\mathrm{SiO}_{2}, \mathrm{ThO}_{2}, \mathrm{TiO}_{2}$ (d). 1 - end of the added layer of $\mathrm{TiO}_{2}$ and begining of the layer $\mathrm{A}$ for the first period; 2 - end of the layers $\mathrm{A}$ and begining of the layer $\mathrm{B} ; 3$ - end of the layers $B$ and begining of the layer $C ; 4$ - end of the layers $C$ and begining of the layer B; 5 - end of the layers B and begining of the layer A; 6 - end of the layers A for the first period.

The transmission spectra of obtained multilayer structures with the periods (1)-(4) are shown in Fig. 4. They practically coincide with theoretical calculations in which the data on the dispersion of optical constants (Fig. 2) are used. The following differences of experimental spectra and theoretical predictions (a case of absence of the dispersion, see Fig. 1) are observed.
First, sharp decrease of transmission in the shortwave range (less than $400 \mathrm{~nm}$ ) because of strong absorption in $\mathrm{TiO}_{2}$ layers. Second, occurrence in some structures of small transmission failures (to $5-10 \%$ ) on wavelengths of high reflection bands of 4-th and 6-th orders connected to infringement of the optimum ratio of refractive indecies (5)-(8). Theoretical curves of total 


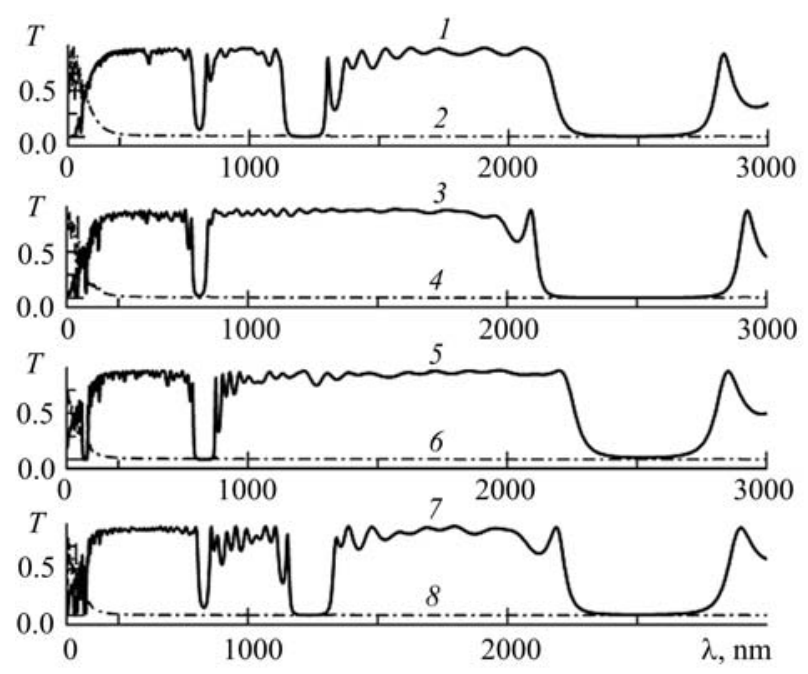

Fig. 4. Experimental spectral dependences of transmission (1, $3,5,7)$ and the theoretical ones of absorption $(2,4,6,8)$ for multilayer structures $\mathrm{S}(\mathrm{ABCCCCCCBA}){ }^{10} \mathrm{~S}_{0}-1,2$; $\mathrm{S}(\mathrm{AABCCCCBAA}){ }^{10} \mathrm{~S}_{0}-3,4 ; \mathrm{S}(\mathrm{ABBBCCBBBA}){ }^{10} \mathrm{~S}_{0}-5,6$; $\mathrm{S}(A A A B C C B A A A){ }^{10} \mathrm{~S}_{0}-7,8 . \mathrm{S}$ - substrate of $\mathrm{SiO}_{2} ; \mathrm{S}_{0}-$ air; $\mathrm{A}-\mathrm{SiO}_{2} ; \mathrm{C}-\mathrm{TiO}_{2} ; \mathrm{B}-\mathrm{HfO}_{2}(1,2), \mathrm{Pr}_{6} \mathrm{O}_{11}(3-6), \mathrm{ThO}_{2}(7,8)$.

absorption in researched structures are shown in Fig. 4. It is obvious that the more total thickness of $\mathrm{TiO}_{2}$ layers, the more total absorption of structures.

\section{Conclusions}

Thus, presence of the dispersion of optical constants results in impossibility of strict performance of the optimum ratio of layer refractive indecies as caused by possible occurrence of trasmission failures at wavelengths of separate supported high reflection bands. To reduce this effect, it is necessary to achieve the feasibility of the optimum ratio of refractive indecies at the wavelength of the middle of the range for the formed transmission band. Besides, at this wavelength optical thicknesses of separate layers in the period should be multiple. One of the areas to use the considered multilayer systems is creation of various protective means capable to avoid influence of optical radiation at multiple wavelengths, and also to use them as mirrors in laser resonators for the IR-range with longitudinal pumping.

\section{References}

1. H.A. Macleod, Thin-film optical filters. Adam Hilger Ltd., Bristol, p.1-332 (2001).

2. Yu.A. Pervak, I.V. Fekeshgazi, Optical properties of unthicknesses multilayer coatings with symmetrical periods // Quant. Electron. (Kiev) 46, p.8-11 (1994).

3. A. Thelen, Multilayer filters with wide transmittance bands // J. Opt. Soc. Amer. 53(11), p. 1266-1270 (1963).

4. M.P. Lisitsa, S.V. Orlov, Yu.A. Pervak, I.V. Fekeshgazi, Multilayer coatings with supporting of two adjacent zone of high reflection // J. Appl. Spectr. 47(2), p.283-284 (1987).

5. A. Thelen, Multilayer filters with wide transmittance bands, II // J. Opt. Soc. Amer. 63 (1), p.65-68 (1973).

6. A. Thelen, Interference filter // Patent FRG N 2357593 (1975).

7. Yu.N. Markov, E.A. Nesmelov, A.G. Gusev, Syntesis of contrast band interference filters // Optika $i$ Spektroskopiya 53(6), p.1091-1096 (1982) (in Russian).

8. V.Yu. Pervak, Spectral properties of interference filters formed miltiple repeating of three element blocks // Opt. Journ. 70(10), p.91-95 (2003).

9. V.Yu. Pervak, Yu.A. Pervak, Syntesis of wideband interference filters II. Suppress of high reflection bands of 4, 5 and 6 orders // Optika i Spektroskopiya 96(3), p. 461-466 (2004) (in Russian).

10. V.Yu. Pervak, Yu.A. Pervak. Suppress of high reflection bands of 4, 5 and 6 orders in interference filters // Journ. Appl. Spectr. 70(5), p.617-621 (2003).

11. V.Yu. Pervak, I.V. Fekeshgazi, Yu.A. Pervak, Interference filter // Patent Ukraine № 53265A, Bull. 1 (2003).

12. V.Yu.Pervak, Yu.A. Pervak, Interference filter ABCBA-1R3R456T // Patent Ukraine № 58887A, Bull. 8 (2003).

13. V.Yu. Pervak, Yu.A. Pervak, Interference filter ABCBA-123R456T // Patent Ukraine № 58888A, Bull. 8 (2003).

14. V.Yu. Pervak, Yu.A. Pervak, Band interference filter 567-8 // Patent Ukraine № 58889A, Bull. 8 (2003). 